\title{
Historical Fire Detection of Tropical Forest from NDVI Time-series Data: Case Study on Jambi, Indonesia
}

\author{
Dyah R. Panuju ${ }^{1}$, Bambang H. Trisasongko ${ }^{1}$, Budi Susetyo ${ }^{2}$, \\ Mahmud A. Raimadoya ${ }^{3} \&{\text { Brian G. } \text { Lees }^{4}}^{4}$ \\ ${ }^{1}$ Department of Soil Science and Land Resources, Bogor Agricultural University. Jalan \\ Meranti. Bogor 16680. Indonesia. Email: d.panuju@ hotmail.com \\ ${ }^{2}$ Department of Statistics, Bogor Agricultural University. Bogor 16680. Indonesia. \\ ${ }^{3}$ Department of Civil and Environmental Engineering, Bogor Agricultural University. \\ Bogor 16680. Indonesia. \\ ${ }^{4}$ School of Physical, Environmental and Mathematical Sciences, University of New \\ South Wales at Australian Defence Force Academy. Northcott Drive, Canberra ACT \\ 2600. Australia

\begin{abstract}
In addition to forest encroachment, forest fire is a serious problem in Indonesia. Attempts at managing its widespread and frequent occurrence has led to intensive use of remote sensing data. Coarse resolution images have been employed to derive hot spots as an indicator of forest fire. However, most efforts to verify the hot spot data and to verify fire accidents have been restricted to the use of medium or high resolution data. At present, it is difficult to verify solely upon those data due to severe cloud cover and low revisit time. In this paper, we present a method to validate forest fire using NDVI time series data. With the freely available NDVI data from SPOT VEGETATION, we successfully detected changes in time series data which were associated with fire accidents.
\end{abstract}

Keywords: SPOT VEGETATION; outlier detection; X12-ARIMA.

\section{Introduction}

Forest fires in Indonesia have a wide impact on both humans and the environment. Fire accidents are a major cause of loss of life and severe degradation of air quality which, in turn, affect human health [1]. In the case of the islands of Sumatra and Kalimantan, cross-border smoke often raises political problems between Indonesia and Malaysia and Singapore. Fire is also one of the main factors causing environmental degradation. It causes fragmentation of the forest cover leading to greater risk during the subsequent fire episodes. At the global scale, frequent forest fires are one of the major contributors to a change in carbon balance [2] and have become a key component in climate change.

Efforts to combat forest fire are underway. A special task force to deal with forest fire has been assembled. In a number of major crises, it has been assisted

Received October $4^{\text {th }}, 2009$, Revised February $2^{\text {nd }}, 2010$, Accepted for publication February $5^{\text {th }}, 2010$. 
by ASEAN fire fighters to reduce the trans-national impact. With very extensive areas affected, remote sensing provides invaluable data for both decision makers and local fire fighters. Remote sensing also offers continuous monitoring for tactical decision making [3].

Fire frequently occurs in both tropical and boreal forests. In boreal forests, several episodes of large forest fires have been recorded which have raised the importance of time-series assessments [4]. In tropical forests, major forest fires have been associated with the El Nino Southern Oscillation (ENSO) which is associated with extreme drought making them regional, rather than local, phenomena [5]. It was also worsened by human activity encroached not only to primary forest but also preserved forest that can lead to carbon leaks from tropical area. Indeed, to detect and prove tropical forest encroachments, particularly if triggered by fire, are difficult due to frequent precipitation which inhibits fire. Monitoring by remote sensing has been difficult because of limited revisit time of high resolution sensors. Although many hot spot data have been produced by many satellites, but it has been limitedly proven as real fire spot. As fire is very dynamic, time series data are required to comprehensively assess the progress of the phenomenon. Coarse resolution remote sensing data offer possibilities to provide time-series data over extensive areas.

Time-series remotely sensed data have been used in other applications. In meteorology, Liu et al. [6] presented a method to map evapotranspiration using time-series data. In agriculture, using the AVHRR sensor, Schwartz et al. [7] demonstrated the capability of remote sensing data to derive information on the start of the spring growing season to quantify the influence of climate change. Another study, Julien et al. [8], emphasized the importance of long time-series remote sensing data to assess the contribution of volcanic aerosols to the vegetation dynamics of the European continent. Because of the return frequency, Moderate Resolution Imaging Spectrometer (MODIS) data has been preferred for many of these land-based remote sensing applications such as forest phenology $[9,10]$.

In the case of forest fire, time-series data have been employed for various purposes. Mapping of the burned scar has been the most common application. Coarse remotely sensed images have been used for this purpose including both NOAA AVHRR and MODIS [11]. Generally, forest fire has been assessed through time-series composite techniques. This minimises radiometric problems such as effects of local atmospheric disturbances. Present time-series compositing algorithms were derived from the Maximum Value Composite (MVC) of Holben [12]. However, the MVC method has been found to be less reliable for semi arid areas which generally have lower NDVI values [11]. 
While previous efforts have concentrated on mapping fire-affected areas, it is also important to determine the time of the accident. In Indonesia, this information is important for law enforcement as massive slash-and-burn practices are illegal, in particular for plantation companies. Goetz et al. [13] showed NDVI patterns of burned and unburned areas which were particularly useful for studying post-burn recovery. According to their report, fire had a dramatic effect in the NDVI time series data, suggesting that the technique could be used to confirm the time of fire initiation, especially from hot spot data.

Obviously, the NDVI is not a perfect choice to the problem. Viovy [14] showed NDVI dependencies on atmospheric disturbances due to volcanic eruption. Application of NDVI on forage prediction was also found limited. Other vegetation indices have been invented and showed better performance [15]. Nonetheless, applying those newly-developed vegetation indices such as Enhanced Vegetation Index (EVI) designed from MODIS data in time series analyses is usually limited due to availability of the dataset itself. On the other hand, NDVI provides long-term data capture suitable for time series analyses. Recent reports still have commented the usefulness of NDVI time series data $[16,17]$.

Extending the work of Goetz et al. [13], and to evaluate the quantitative determination of fire initiation, we carried out an analysis of time series data. We employed the X12-ARIMA algorithm to provide the date automatically from the NDVI data of SPOT VEGETATION made available by Vlaamse Instelling voor Technologisch Onderzoek (VITO). The use of freely available data is a significant benefit for the preliminary screening for higher resolution data capture for operational purposes.

\section{Time Series Analysis}

Time series data are a chain of observations acquired over time and often contain a constant time lag. The observations can be single variable or multivariable which, in remote sensing terminology, corresponds to single and multiple data respectively. The data are not limited to raw electromagnetic signals, but also cover derivative information such as spectral indices or thematic data.

Assumptions in time series analysis are homogeneity of variance and data stationarity. Homogeneity is detected as being a constant variance in time, while stationarity can be defined as a condition when there are no systematic changes in the mean of the series and without periodic shift in variances [18]. 
Unfortunately, the stationary condition occurs rarely and therefore a differencing technique is often used to handle non stationary series.

Common modelling on single variable time series has exploited the Autoregressive Moving Average (ARMA) or the Autoregressive Integrated Moving Average (ARIMA) modelling philosophies introduced by Box and Jenkins [19]. While the ARIMA is useful for assessing time series data, it suffers from the effects of seasonality which is naturally found in many circumstances. In order to accommodate this, the X procedure has been used as an alternative procedure for seasonal adjustment.

The combination (X11-ARIMA) has been introduced to compensate for the lack of an explicit model functional to the series and to minimise the unsmoothed first and last observation of the series [20]. Later, the model was updated to X12-ARIMA by Findley et al. [21]. Following the Box-Jenkins notation, a seasonality-induced ARIMA model is represented as $(\mathrm{p}, \mathrm{d}, \mathrm{q})(\mathrm{P}, \mathrm{D}, \mathrm{Q})$. Notations $\mathrm{p}$ and $\mathrm{P}$ point to the ordinary and seasonal autoregressive respectively, while $\mathrm{q}$ and Q indicate the properties of moving averages. Respectively, $\mathrm{d}$ and D specify ordinary and seasonal differences in from the primary series. The use of X11ARIMA, the previous version of seasonal ARIMA, in environmental research was found, such as on climate research [22].

\section{Outlier Detection}

Outlier detection was an improvement capacity of ARIMA to deal with outrageous evidences during the series. Adya et al. [23] defined outliers or contaminant data as unusual observations that shift from the general pattern of the set. While time series data generally contain outliers, they have been disregarded in many studies. There are only a few which identify the various types of outlier. Fox [24] noted two types of outlier, namely type I and type II. Type I relates to the state where a single observation can be influenced by gross error. Another type is used when the influence is observed not only on a specific observation, but continues onto the succeeding observation. Later, type I and type II were identified by Chang et al. [25] as additive (AO), and innovational (IO), outliers respectively. If a series is under consideration, a single observation in the series can be either $\mathrm{AO}, \mathrm{IO}$ or neither. Iterative procedures to test for the presence of outliers have been presented [25-27] under the ARIMA framework. In addition to these types of outlier, Tsay [26] presented the level shift (LS), which was then divided into the level change (LC) or permanent change (PC) and transient change (TC) outliers. The most recent paper by Kaiser and Maravall [28] established another type of outlier namely the seasonal level shift (SLS). 
X12-ARIMA automodelling can assess the four types of outliers discussed by Chen and Liu [29], namely the innovational outlier (IO), the additive outlier (AO), the transient change (TC) and the level shift (LS). To detect the presence of outliers in time series data, the residual of the series is determined. Residuals contain data unexplained by the model. The assumption of the time series stochastic model is the homogeneity of variance and stationarity of data. The fitted model is evaluated by computing the minimum mean square error, while the stationarity is evaluated from the pattern of the corresponding residuals. Chen and Liu [29] presented equations to compute residuals for the outliers.

In the case of forest fire hot spots, the model should accommodate the detection of multiple outliers. This is due to the high probability of re-burn in the same location related to time lag between estate preparation (land clearing) and canopy closing. Time lag could be a year in case of timber plantation [30]. Hence, the ARMA process can be represented as follows

$$
\mathrm{Y}_{\mathrm{t}}^{*}=\sum_{\mathrm{j}=1}^{\mathrm{m}} \omega_{\mathrm{j}} \mathrm{L}_{\mathrm{j}}(\mathrm{B}) \mathrm{I}_{\mathrm{t}}\left(\mathrm{t}_{\mathrm{j}}\right)+\frac{\theta(\mathrm{B})}{\varphi(\mathrm{B}) \alpha(\mathrm{B})} \mathrm{a}_{\mathrm{t}}
$$

with the error forecast as

$$
\hat{e}_{t}=\sum_{j=1}^{m} \omega_{j} \pi(B) L_{j}(B) I_{t}\left(t_{j}\right)+a_{t}
$$

where $\theta, \alpha, \varphi$ are polynomials of $B ; \quad \varphi(B)=1-\varphi_{1}(B)-\ldots-\varphi_{p}(B)$ and $\theta(B)=\left(1-\theta_{1} B-\ldots-\theta_{p} B^{p}\right)$ are stationary; and $I_{t}\left(t_{j}\right)$ serves as indicator for the presence of outliers and is a binary number where equals to 0 if $t \neq t_{1}$ and 1 otherwise. $\omega$ represents amount and pattern of outlier respectively, while $a_{t}$ is presumed to be a sequence of independent variable with mean 0 and variance $\sigma_{\mathrm{a}}^{2}$.

Taken into account the seasonal pattern of the data, the X12-ARIMA [21] estimates regression ARIMA models of order $(\mathrm{p}, \mathrm{d}, \mathrm{q})(\mathrm{P}, \mathrm{D}, \mathrm{Q})$ for $\mathrm{Y}_{\mathrm{t}}$ as

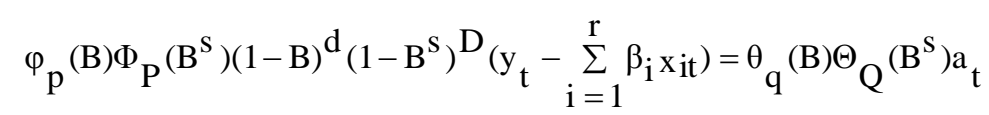

where $\mathrm{s}$ is the length of seasonal period; $\mathrm{s}=12$ represents 12 month of an annual observation, while $\mathrm{p}, \mathrm{d}, \mathrm{q}$ and $\mathrm{P}, \mathrm{D}, \mathrm{Q}$ denotes numbers of first and seasonal times of autoregressive, difference and moving average. The polynomials 
$\varphi_{\mathrm{p}}(\mathrm{z}), \Phi_{\mathrm{P}}(\mathrm{z}), \theta_{\mathrm{q}}(\mathrm{z}), \Theta_{\mathrm{Q}}(\mathrm{z})$ with degrees of $\mathrm{p}, \mathrm{P}, \mathrm{q}, \mathrm{Q}$ respectively have a constant term equal to 1 .

\section{$4 \quad$ Methodology}

The research was carried out around the Berbak National Park in eastern wetland of Jambi province, one of three Ramsar reserves in Indonesia (Figure 1). The area has suffered from logging and frequent fires for land clearing, particularly in the dry season [31].

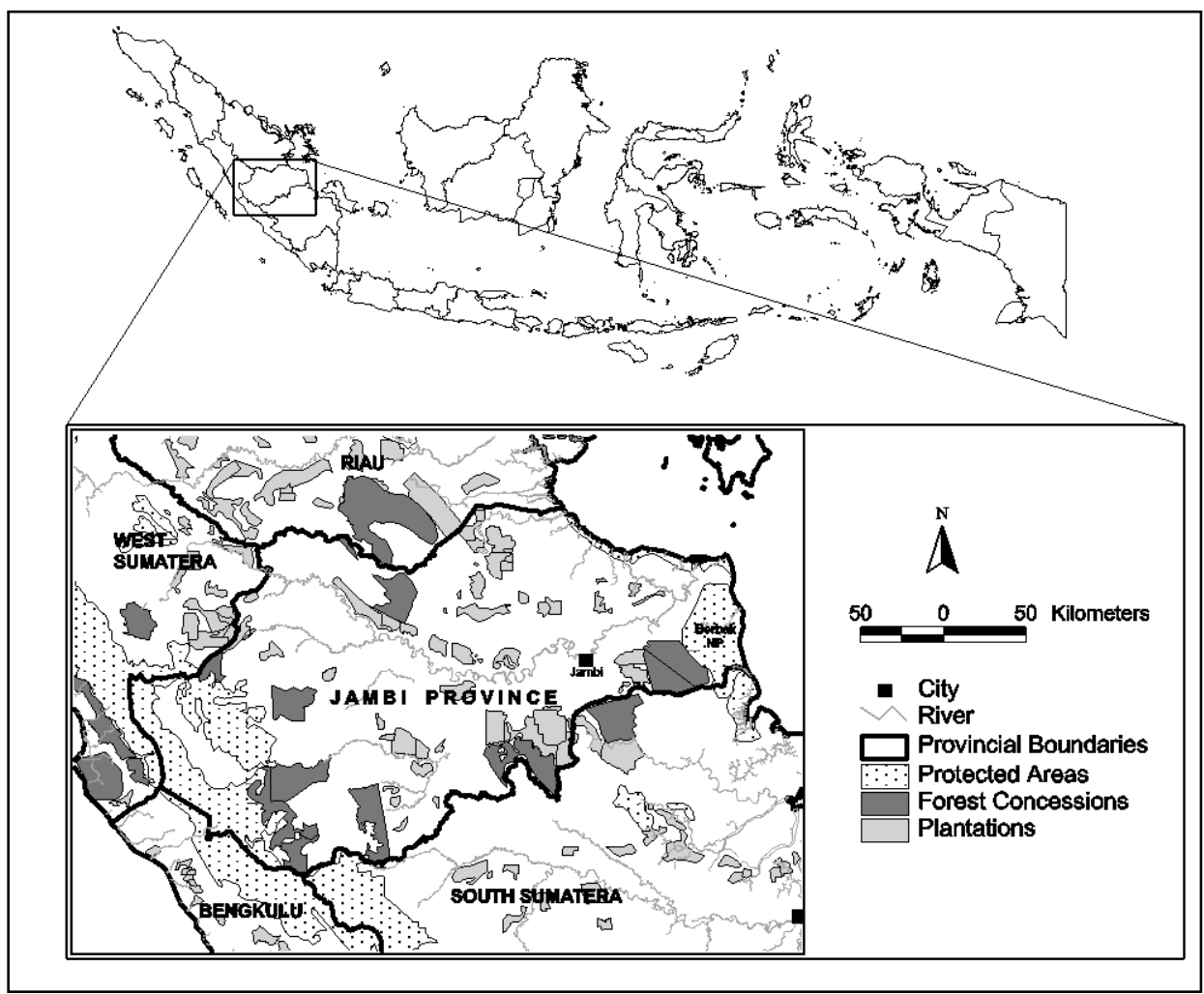

Figure 1 Site location. Dark and light polygons represent forest concessions and plantations respectively. The Berbak NP is located on eastern coastal region.

The study made use of both medium and coarse remote sensing data. Landsat ETM, as medium resolution data, provided actual fire data. We selected a Landsat ETM scene of Jambi (path/row 125-061) acquired on 1 September 1999 which showed a large active fire. It is difficult to have fire evidence data and to capture actual fire in tropical region on image (such as Landsat ETM) 
due to cloud cover, therefore the data was a valuable evidence of fire for a reference on further analysis. No radiometric adjustment was applied, mainly due to the lack of meteorological data. Hotspot data collected from the Indonesian Institute of Space and Aeronautics was also employed for completing and cross checking the evidence. The hotspot data were produced from NOAA-AVHRR from some platforms including NOAA12, 15 and 16. Several techniques for burn scar delineation can be found in the literature, nevertheless we found a paper by Koutsias et al. [32] particularly interesting. The paper discussed the application of the Intensity-Hue-Saturation transformation on specific colour composite image. The technique proved useful, taking advantage of the fast computation of intensity, hue and saturation components from a red-green-blue image composite.

Using the fire extent derived from the medium resolution image, the burned area was gridded to obtain several samples as the main locations for time series analysis. We used NDVI data of SPOT VEGETATION from the VITO website. The NDVI data were distributed as 10-day composite images, hence there were three for each month and the first NDVI $\left(\mathrm{NDVI}_{1}\right)$ data represented a time span between day 1 and day $10, \mathrm{NDVI}_{2}$ denoted day 11 to day 20, and $\mathrm{NDVI}_{3}$ symbolized day 21 to day 30 of the month. To ease extraction of the NDVI time series at each sample point, we stacked all NDVI data taken between 1 April 1998 and 21 February 2005. Due to the limits of the ARIMA model, a monthly aggregation was carried out. To do this we selected the approaches presented by Jia et al. [33]. The monthly average (MA) and monthly peak (MP) were calculated using following equations.

$$
\begin{aligned}
& \mathrm{MA}_{\mathrm{NDVI}}=\operatorname{mean}\left(\mathrm{NDVI}_{1}, \mathrm{NDVI}_{2}, \mathrm{NDVI}_{3}\right) \\
& \mathrm{MP}_{\mathrm{NDVI}}=\max \left(\mathrm{NDVI}_{1}, \mathrm{NDVI}_{2}, \mathrm{NDVI}_{3}\right)
\end{aligned}
$$

We employed Demetra 2.04 for X12-ARIMA modelling and to detect outliers. The software was developed by Eurostat and has been used mainly for finance and monetary applications. The automodelling provided option to choose the appropriate model for univariate series analysis with several adjustments including: (1) pretests for a logarithm transformation (multiplicative/ additive modelling); (2) a mean correction (if necessary); (3) a new ARIMA model identification/ selection and estimation; (4) pre-tests for Easter and one of four different trading day effects (including country-specific holidays); (5) an automatic detection and correction for outliers over the whole time series length; (6) an interpolation of missing observations; (7) an ARIMA forecast at the end of the series; and (8) an automatic decomposition. To test our results, we compared detected outliers with available hotspot data from the Indonesian Institute of Space and Aeronautics (LAPAN). To avoid misregistration over 
various remote sensing data sources, we also considered hotspots around the site at a distance of 0.02 degree.

\section{$5 \quad$ Results and Discussion}

\subsection{Fire delineation from Landsat ETM data}

The IHS transform is a simple image transformation which has been used for many applications including multi-resolution data fusion and enhancement of terrain for interpretation. While those applications require a two-way transformation (RGB to IHS and back to RGB), the thematic extraction of Koutsias et al. [32] only requires a forward transform. Dealing with multispectral images such as Landsat, the key step to extract burn scars is to find suitable band combinations. Koutsias et al. [32] suggested bands of 7, 4, and 1 for the best discrimination. In this study, we used that suggested combination and compared it to a combination of 7-4-2. Figure 2 presents a Landsat colour composite (RGB 542) and the hue components after the IHS transform. The Landsat image coincidentally captured a large active fire along with associated smoke. The burnt area is located in the centre of the image surrounded by various land use types including fragmented forests, clear cut and agricultural fields (Figure 2a).

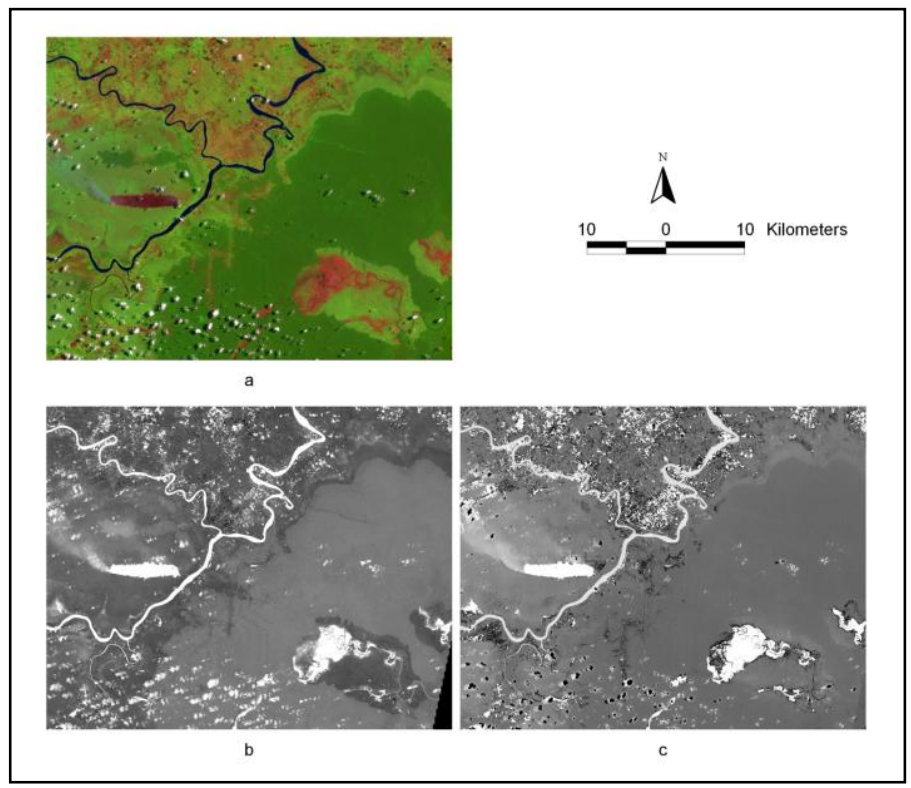

Figure 2 Colour composite image (a) in comparison with hue components of RGB 7-4-1 (b) and RGB 7-4-2 (c). 
From the composite image, the area apparently was burned several times over a short period of time. Slightly different levels of reddish are shown which indicate different intensities of burning or different times of slash-and-burn. Considering that frequent downpours in the eastern Jambi provinces can inhibit fire spread, it can be estimated that attempts to burn started before August 1999. We noticed a good agreement between visual analysis and derived hue images. In general both hue images present clear discrimination of the burn scar and its surrounding land cover. Nonetheless, the hue component derived from 7-4-2 composite is shown in slightly lower contrast (Figure 2c). In the image, both burned area and bare soil are presented in a similar tone, which leads to difficulty in the automated delineation of the burned surface. In general, our result was in agreement with the conclusion of Koutsias et al. [32] that a combination of 7-4-1 produced a better discrimination than of 7-4-2. The result of IHS transform was used to delineate burn area which covered about 1342 hectares. The area was then gridded and we obtained 20 sample series for X-12 ARIMA modelling.

\subsection{ARIMA Automodeling of Tropical Forest}

The seasonal adjustment automodelling was set up for selecting, in order, the autoregressive, differencing, and moving average respectively both on ordinary and seasonal series of data [34]. The model used was the economic application in which Easter and trading day components were taken into account. This model had to be adapted to remove those unnecessary components as they had no meaningful relationship to vegetation greenness represented by NDVI. Figure 3 shows time series data of 20 grid samples. The figure illustrates time series pattern of NDVI which affected by seasonality and therefore difficult to interpret. Visual interpretation solely based on these series might mislead information retrieval if the seasonality is present. Statistical tests such as outlier detection could make a clarification for the problem.

Each sample can be modelled on a particular ARIMA model. Instead of using a particular model, we used automodel ARIMA to allow us more focus on the comparison of NDVI data representation of MA and MP. A particular model has significant meaning in forecasting and it is beyond the scope of this study to explain the difference on each series due to the limitations of our ground information. Models of the 20-samples varied in almost all order of autoregressive, differencing and moving average for both ordinary and seasonal series. Comparisons of the performance of the automodelling processes of MA and MP are presented in Table 1. 


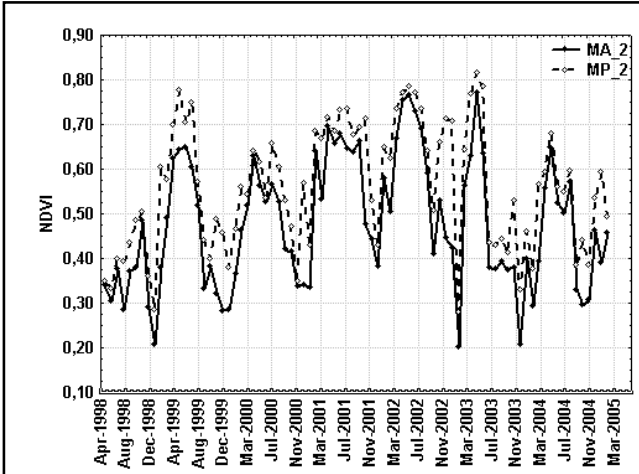

a.

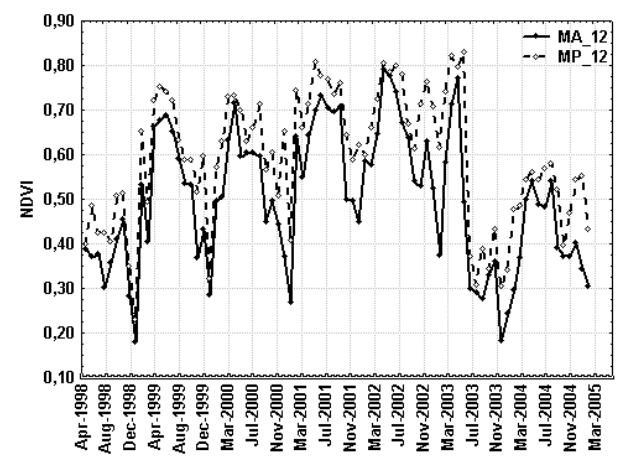

c.

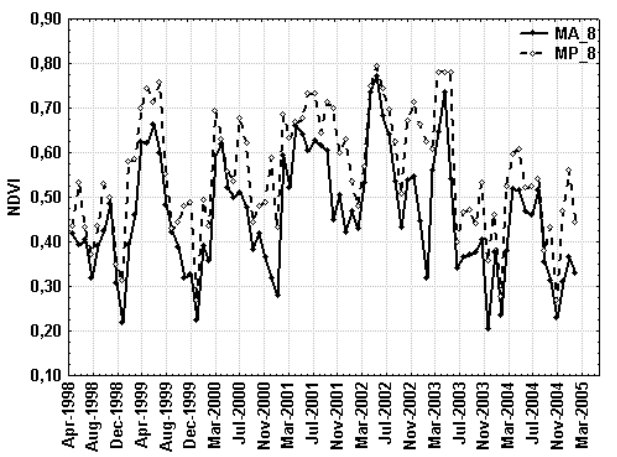

b.

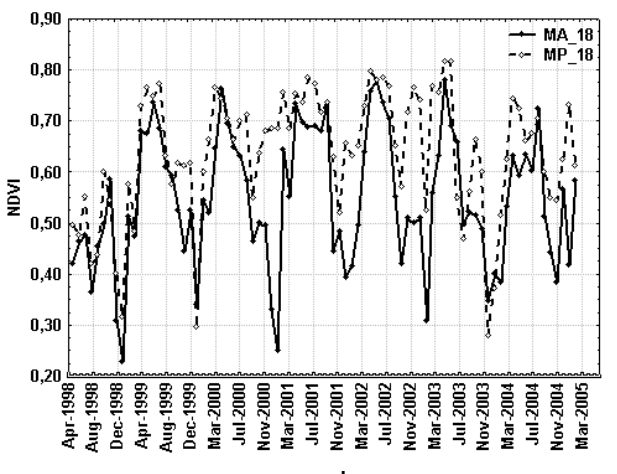

d.

Figure 3 Time series plot of 4 grid samples of MA-NDVI and MP-NDVI.

Table 1 Comparison of some process proceeded on 20 samples of MA and MP on the absolute number and percentage.

\begin{tabular}{ccc}
\hline Statistical Processes & Monthly Average & Monthly Peak \\
\hline Seasonally adjustment & $13(65 \%)$ & $10(50 \%)$ \\
Transformation & $0(0 \%)$ & $0(0 \%)$ \\
Mean correction & $20(100 \%)$ & $20(100 \%)$ \\
Missing observations & $0(0 \%)$ & $0(0 \%)$ \\
Seasonality "significant" & $20(100 \%)$ & $11(55 \%)$ \\
Forecasting error $>10 \%$ & $6(30 \%)$ & $8(40 \%)$ \\
\hline
\end{tabular}

Table 1 shows similar performances between MA and MP on some processes, particularly in the requirement of data transformation, mean correction and detection of missing observations for the samples. A significant difference of MA and MP was shown in the detection of the seasonality effect on each sample series. All MA series had a significant seasonal pattern while only 11 
samples (55\%) of MP series were affected by seasonality. However, seasonality was probably present in the remaining samples of MP. Most of the MA and MP samples were seasonally adjusted allowing proper seasonal pattern identification. All MA samples were seasonally significant which means that all samples were seasonally affected. Another process shown in Table 1 is forecasting error. Slight differences in performance were shown between MA and MP which indicated that 6 samples of MA and 8 samples of MP were not reliable for quantitative analysis. It appears that previous studies by Goetz et al. [13] assumed equal significance of series taken from different sites. It shown here that even if all samples were taken from a similar condition (delimitation of extracted burned areas), different characteristics of series were evident. Different aggregation processes by assigning average or maximum values over specific time frame produced different results. We observed that excessive aggregation would likely generate biased interpretation. Using information from Table 1, we were able to show those differences and hence able to select reliable series.

\subsection{Outlier Extraction}

Particularly on the eastern provinces of Sumatra, slash-and burn techniques have been practiced for land clearing. Hence, a high number of forest fires have been detected in this region. Due to the humid climate and high rainfall, repeated burning has been observed as slash-and-burn activities may be interrupted by the rain. Burning can be continued several days later once the surface has dried. This pattern creates a high frequency of hot spots detected by remote sensing and explains why hot spots occur at the same location.

Detection of forest fire using Landsat data indicated that repeated burning occurred on the test site. Theoretically, the fire created a hiatus in vegetation condition represented on the NDVI time series data where the NDVI values sharply decreased. Since long term observations can contain seasonal patterns [35], that phenomenon should be accounted for during data processing. We assumed that ten-day NDVI or their monthly aggregation data were shown as an annual repeated pattern. Hence outlier detection looked for any hiatus in the data while taking account of the seasonal pattern. The outlier detection offers precise identification of date (or in our case month and year) of fire occurrence, therefore improves on Goetz et al. [13] findings. Results of outlier detection using MA and MP are presented in Table 2.

The Landsat image was used as a point of reference to be compared with detected outliers. Considering slash-and-burn behaviour on the site, we also took into account burning before the date of Landsat acquisition. In this case, we assumed August 1999 and September 1999 as an exact time of burning on 
the site. There appears to be little difference in performance between MA and MP on outlier detection. Many outliers were successfully detected on MA and MP samples. Some were shown to share an identical date with our hot spot database and the Landsat image. We considered a month after outlier date to be identical because the change of greenness due to fire can be detected after the burning, particularly if it was taken place on last ten day of the month.

Table 2 Outlier detection using monthly average and monthly peak data. Outliers in bold indicate in agreement with Landsat image, while in underline in agreement with hot spot data respectively.

\begin{tabular}{|c|c|c|}
\hline $\begin{array}{l}\text { Sample } \\
\text { series }\end{array}$ & MA_NDVI & MP_NDVI \\
\hline Grid1 & Sep-98, Sep-01, Feb-03, Dec-03 & Sep-99, Feb-05 \\
\hline Grid2 & $\begin{array}{c}\text { Sep-98, Aug-99, Oct-01, Feb-03, } \\
\text { Jul-03, Dec-04, Jan-05 }\end{array}$ & $\begin{array}{c}\text { Aug-99, } \\
\text { Dec-02, Feb-03, Jul-03, } \\
\text { Dec-04 }\end{array}$ \\
\hline Grid3 & $\begin{array}{l}\text { Oct-98, Sep-99, Nov-01, Mar-03, } \\
\underline{\text { Aug-03, Jan-05, Feb-05 }}\end{array}$ & 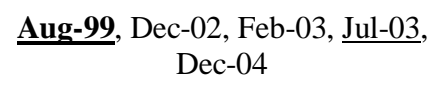 \\
\hline Grid4 & Sep-99, Jul-03, Dec-04, Jan-05 & Sep-99, Feb-03, Jul-03 \\
\hline Grid5 & Feb-99, Mar-01 & $\underline{\mathrm{Jul}-03}$ \\
\hline Grid6 & Feb-99, Feb-00, Feb-03, Jul-03 & Mar-03, Jul-03 \\
\hline Grid7 & Feb-99, $\underline{\text { Jul-03 }}$ & Feb-99, Dec-02, Jul-03, Jun-04 \\
\hline Grid8 & 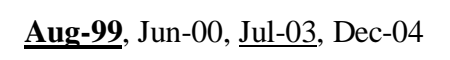 & \\
\hline Grid9 & 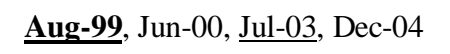 & \\
\hline Grid10 & $\begin{array}{l}\text { Aug-99, Jun-00, Mar-01, Oct-02, } \\
\underline{\text { Jul-03 }} \text {, Dec-03, Jan-05 }\end{array}$ & Jan-01, Jul-03 \\
\hline Grid11 & Sep-99, Jul-03, Dec-03 & $\begin{array}{l}\text { Sep-99, Nov-02, Feb-03, Jul-03, } \\
\text { Dec-03, Dec-04, Jan-05 }\end{array}$ \\
\hline Grid12 & $\underline{\text { Jun-03 }}$ & $\underline{\mathrm{Jul}-03}$ \\
\hline Grid13 & Dec-04 & Jan-99, Dec-04 \\
\hline Grid14 & & Feb-99, Feb-01 \\
\hline Grid15 & & Feb-99, Feb-01, Feb-03, Nov-04 \\
\hline Grid16 & & Feb-99, Jan-00, Feb-01, Dec-03 \\
\hline Grid17 & & Feb-99, Jan-00 \\
\hline Grid18 & & Apr-99, Jan-00, Jul-03, Dec-03 \\
\hline Grid19 & Nov-01, Jun-03 & $\begin{array}{l}\text { Apr-99, Apr-00, Nov-01, Oct-02, } \\
\text { Dec-02, Jul-03, Feb-04, Jan-05 }\end{array}$ \\
\hline Grid20 & & Jan-99, Jan-00, Jul-03 \\
\hline
\end{tabular}


The result shows that the MA and MP contained four series with outliers which were detected coincident with fire detection from Landsat image on August/September 1999 at different sites (Grid 2, 3, 4 and 11). Based on the presumption that significant effect of fire would be manifested on outrageous change on NDVI series, it could be inferred that the corresponding sample was affected by fire. In addition, we obtained seven samples of MA and MP that contained similar dates to detected hot spots (July 2003). There were fifteen samples of MA and fourteen samples of MP which showed good agreement with hotspot data, although these could not be validated due to the lack of additional reference data. Complete hotspots detected in samples area are presented in Table 3.

Table 3 Number of hospots on sample areas (location-1) or \pm 0.02 degree of longitude or latitude (location-2), and their date (month-year).

\begin{tabular}{ccc}
\hline Location & Month & Number of hotspots \\
\hline 1 & Aug-99 & 2 \\
2 & Aug-99 & 6 \\
2 & Sep-02 & 2 \\
1 & Jun-03 & 16 \\
2 & Jun-03 & 35 \\
2 & Jul-03 & 2 \\
2 & Aug-03 & 5 \\
1 & Aug-04 & 1 \\
2 & Aug-04 & 2 \\
2 & Sep-04 & 2 \\
1 & Oct-04 & 2 \\
\hline
\end{tabular}

The results show that we were able to assess the importance of the degree of burn severity. We found that all outliers that coincidentally related to fire had the LS type. According to Tsay [26], this type has long-lasting effect. Vaage [36] noticed that LS showed an impact that permanently shifted the following pattern of the series. LS had also been shown to affect the parameter estimation of ARMA making it inconsistent by changing the mean of the series. Seven outliers of MA (five on August 1999 and two on June 2003) were in agreement with hotspots and fire on the corresponding sites in LS-type. In addition, 9 outliers of MA, including 8 outliers in TC-type and 1 outlier in LS-type were consistent with the hotspot of July and August 2003. Furthermore, 2 outliers of MP were in agreement with hotspot and fire on August 1999 and 13 outliers of 
MP comprised 6 outliers in TC-type and 7 in LS-type was also coincident with hotspot of July 2003 in surrounding site or with a month later of hotspot detected in the site. We did not deal with all types of outliers due to limitations of information to deliver proper explanations.

The result of outlier detection of MA and MP were quite different, however they showed that both achieved their aim. Many outliers could not be precisely linked to burning activity on the corresponding date of hotspot. Some other possible activities, such as logging, can affect a similar change of vegetation greenness. It appears that outlier detection of NDVI series is a potential method to explore historical evidences of vegetative area. Outliers could be a sign that there was an important phenomenon occurred on the specific date. Detailed information should be explored to prove and dig up initial information provided from outlier detection analysis.

\section{Conclusions}

In this paper, the application of outlier detection using X12-ARIMA for exploring historical evidence of forest fire was investigated. It seems that part of Jambi natural forest have been suffering from encroachment since before August 1999 and still continuing. Using IHS transformation, hue image of bands 7-4-1 composite could discriminate clearly burn scars. Mapping of burn scars confirmed active fire encroaching the test site. Image extraction on sample area provided information on the efforts to convert natural forests to cultivated area. Using X12-ARIMA the efforts were detected as outliers on NDVI time series. LANDSAT ETM acquisition date was identified in the outlier list. Four outliers were detected on MA and two on MP samples which coincide with hotspots data and LANDSAT image. In addition, three MA and three MP samples were able to detect outliers which correspond with a month after fire captured on image. Apparently, sensitivity on outlier detection using MA and MP showed indifferent. Using auto-modelling of ARIMA, we showed a quantitative analysis of selected sites. We extended the approach outlined by Goetz et al. [13] by providing the date of the events from time series databases of NDVI. Research of Goetz et al. [13] tent to disregard this selection and generally assumed that all sample sets are equally reliable.

Despite recent availability of many vegetation indices, the NDVI time series data were shown useful, mainly due to their long-term accessibility. Nonetheless, we urge further assessments to exploit other indices such as the Enhanced Vegetation Index (EVI). Comparison of the use of different vegetation indices may provide a more reliable index for detection of historical changes in vegetated areas. Vegetation indices of MODIS (Moderate Resolution Imaging Spectro-radiometer) should also be investigated for better 
understanding due to its detailed scale. Improving capacity on ARIMA modelling in analysing ten days data would also enhance precision in outlier detection.

\section{Acknowledgements}

We would like to thank VITO, Belgium for their support obtaining SPOTVEGETATION dataset and BPPS-DIKTI, Indonesian Ministry of Education for the scholarship. Part of the references was obtained from the Library of Universita degli Studi di Roma - Tor Vergata and we are grateful for Profs. Domenico Solimini and Leila Guirerro assistances.

\section{$7 \quad$ References}

[1] Mott, J.A., Mannino, D.M., Alverson, C.J., Kiyu, A., Hashim, J., Lee, T., Falter, K. \& Redd, S.C., Cardiorespiratory Hospitalizations Associated with Smoke Exposure during the 1997 Southeast Asian Forest Fire, International Journal of Hygiene and Environmental Health, 208, 75-85, 2005.

[2] Harden, J.W., Trumbore, S.E., Stocks, B.J., Hirsch, A., O’Neill, K.P. \& Kasischke, E.S., The Role of Fire in the Boreal Carbon Budget, Global Change Biology, 6 (Supp 1), 174-184, 2000.

[3] Keramitsoglou, I., Kiranoudis, C.T., Sarimveis, H., Sifakis, N., A Multidiscplinary Decision Support System for Forest Fire Crisis Management, Environmental Management, 33, 212-225, 2004.

[4] Amiro, B.D., Orchansky, A.L., Barr, A.G., Black, T.A., Chambers, S.D., Chapin III, F.S., Goulden, M.L., Litvak, M., Liu, H.P., McCaughey, J.H., McMillan, A. \& Randerson, J.T., The Effect of Post-Fire Stand Age on The Boreal Forest Energy Balance, Agricultural and Forest Meteorology, 140, 41-50, 2006.

[5] Fuller, D.O. \& Murphy, K. The ENSO-Fire Dynamic in Insular Southeast Asia, Climatic Change, 74, 435-455, 2006.

[6] Liu, J., Chen, J.M. \& Cihlar, J., Mapping Evapotranspiration Based on Remote Sensing: An Application to Canada's Landmass, Water Resource Research, 39, doi:10.1029/2002WR001680, 2003.

[7] Schwartz, M.D., Reed, B.C. \& White, M.A., Assesing Satellite-Derived Start-of-Season Measures in The Conterminous USA, International Journal of Climate, 22, 1793-1805, 2002.

[8] Julien, Y., Sobrino, J.A. \& Verhoef, W., Changes in Land Surface Temperatures and NDVI Values Over Europe Between 1982 and 1999, Remote Sensing of Environment, 103, 43-55, 2006. 
[9] Ahl, D.E., Gower, S.T., Burrows, S.N., Shabanov, N.V., Myneni, R.B. \& Knyazikhin, Y, Monitoring Spring Canopy Phenology of A Deciduous Broadleaf Forest Using MODIS, Remote Sensing of Environment, 104, 88-95, 2006.

[10] Xiao, X., Hagen, S., Zhang, Q., Keller, M., Moore III, B., Detecting Phenology of Seasonally Moist Tropical Forests in South America with Multi-Temporal MODIS Images, Remote Sensing of Environment, 103, 465-473, 2006.

[11] Chuvieco, E., Ventura, G., Martin, M.P. \& Gomez, I., Assessment of Multitemporal Compositing Techniques of MODIS and AVHRR Images for Burned Land Mapping, Remote Sensing of Environment, 94, 450462, 2005.

[12] Holben, B.N., Characteristics of Maximum-Value Composite Images from Temporal AVHRR Data, International Journal of Remote Sensing, 7, 1417-1434, 1986.

[13] Goetz, S.J., Fiske, G.J. \& Bunn, A.G., Using Satellite Time-Series Data Sets to Analyze Fire Disturbance and Forest Recovery Across Canada, Remote Sensing of Environment, 101, 352-365, 2006.

[14] Viovy, N., Automatic Classification of Time Series (ACTS): A New Clustering Method for Remote Sensing Time Series, International Journal of Remote Sensing, 21, 1537-1560, 2000.

[15] Moleele, N., Ringrose, S., Arnberg, W., Lunden, B. \& Vanderpost, C., Assessment of Vegetation Indexes Useful for Browse (Forage) Prediction In Semi-Arid Rangelands, International Journal of Remote Sensing, 22, 741-756, 2001.

[16] Bradley, B.A., Jacob, R.W., Hermance, J.F. \& Mustard, J.F., A Curve Fitting Procedure to Derive Inter-Annual Phenologies from Time Series of Noisy Satellite NDVI Data, Remote Sensing of Environment, 106, 137145, 2007.

[17] Busetto, L., Meroni, M. \& Colombo, R., Combining Medium and Coarse Spatial Resolution Satellite Data to Improve The Estimation of Sub-Pixel NDVI Time Series, Remote Sensing of Environment, 112, 118-131, 2008.

[18] Wei, W.W.S., Time Series Analysis: Univariate and Multivariate Methods, Pearson/Addison-Wesley, Boston, 2006.

[19] Box, G.E.P. \& Jenkins, G.M., Time Series Analysis: Forecasting and Control, Holden-Day, San Francisco, 1976.

[20] Dagum, E.B., The X-11-ARIMA Seasonal Adjustment Method, Statistics Canada, 1980.

[21] Findley, D.F., Monsell, B.C., Bell, W.R., Otto, M.C. \& Chen, B-C., New Capabilities and Methods of the X-12-ARIMA Seasonal-Adjustment 
Program, Journal of Business and Economic Statistics, 16, 127-152, 1998.

[22] Pezzulli, S., Stephenson, B. \& Hannachi, A., The Variability of Seasonality, Journal of Climate, 18, 71-88, 2005.

[23] Adya, M., Collopy, F., Armstrong, J.S. \& Kennedy, M., Automatic Identification of Time Series Features for Rule-Based Forecasting, International Journal of Forecasting, 17, 143-157, 2001.

[24] Fox, A.J., Outliers in Time Series, Journal of Royal Statistical Society Series B, 34, 350-363, 1972.

[25] Chang, I., Tiao, G.C. \& Chen, C., Estimation of Time Series Parameters in The Presence of Outliers, Technometrics, 30, 193-204, 1988.

[26] Tsay, R.S., Outliers, Level Shifts and Variance Changes in Time Series, Journal of Forecasting, 7, 1-20, 1988.

[27] Chen, C. \& Liu, L.M., Joint Estimation of Model Parameters and Outlier Effects in Time Series, Journal of American Statistical Association, 88, 284-297, 1993a.

[28] Kaiser, R. \& Maravall, A., Seasonal Outliers in Time Series, Banco de Espana, Madrid, Spain, 2001.

[29] Chen, C. \& Liu, L.M., Forecasting Time Series with Outliers, Journal of Forecasting, 12, 13-35, 1993b.

[30] Lorenzo, E.P. \& Munoz, C.P., Land Clearing without Fire: A Model for Preparing Timber Plantation Development in Indonesia, in Proceeding of Workshop on Peatland fire in Sumatera: problems and solutions, Palembang, 10-11 December 2003.

[31] Stolle, F., Chomitz, K.M., Lambin, E.F. \& Tomich, T.P., Land Use and Vegetation Fires in Jambi Province, Sumatera, Indonesia, Forest Ecology and Management, 179, 277-292, 2003.

[32] Koutsias, N., Karteris, M. \& Chuvieco, E., The Use of Intensity-HueSaturation Transformation of Landsat-5 Thematic Mapper Data for Burned Land Mapping, Photogrammetric Engineering and Remote Sensing, 66, 829-839, 2000.

[33] Jia, G.J., Epstein, H.E. \& Walker, D.A., Spatial Characteristics of AVHRR-NDVI along Latitudinal Transects in Northern Alaska, Journal of Vegetation Science, 13, 315-326, 2002.

[34] Gomez, V. \& Maravall, A., Program TRAMO and SEATS: Instructions for the User, Beta Version, Banco de Espana, Madrid, Spain, 1997.

[35] Herrmann, S.M., Anyamba, A. \& Tucker, C.J., Recent Trends in Vegetation Dynamics in The African Sahel and Their Relationship to Climate, Global Environmental Change, 15, 394-404, 2005. 
[36] Vaage, K., Detection of Outliers and Level Shifts in Time Series: An Evaluation of Two Alternative Procedures, Journal of Forecasting, 19, 23-37, 2000. 